\title{
Functional and structural insights on self-assembled nanofiber-based novel antibacterial ointment from antimicrobial peptides, bacitracin and gramicidin $\mathrm{S}$
}

\author{
Santi M Mandal ${ }^{1}$, Anupam Roy ${ }^{1}$, Denial Mahata ${ }^{1}$, Ludovico Migliolo ${ }^{2}$, Diego O Nolasco ${ }^{2}$ and \\ Octavio L Franco ${ }^{2}$
}

A novel antibacterial ointment using bacitracin, specific for Gram-positive bacteria, and gramicidin S, a highly toxic antibacterial peptide, was here developed showing broad-spectrum antibacterial activities against pathogenic strains with less toxicity after self-assembly into nanofiber structures. Such structures were confirmed with scanning electron microscopy and CD analyses. In addition, in silico studies using docking associated with molecular dynamics were carried out to obtain information about fiber structural oligomerization. Thus, the bacitracin and gramicidin S-based self-assembled nanopeptide ribbon may be a successful ointment formulation for bacterial infection control.

The Journal of Antibiotics (2014) 67, 771-775; doi:10.1038/ja.2014.70; published online 4 June 2014

\section{INTRODUCTION}

Development of new classes of antimicrobial agents and alternative treatments $^{1,2}$ has now become a key issue to control the emergence of antibiotic-resistant bacteria. Besides, the focus has switched from developing an antibiotic with a specific function to a more potent one, with a mixture of components or developing new structures from already existing antibiotics to make them capable of regulating a wide range of Gram-positive and Gram-negative bacteria. In the past few decades, strategies like applications of naturally occurring antimicrobial peptides (AMPs), ${ }^{3}$ combined administration of antibiotic agents ${ }^{4-6}$ and structural modification of antibiotics are extensively studied. Structural modification of AMPs by a self-assembly mechanism enhances the spectrum of the AMPs. ${ }^{7}$ The advantage of a self-assembly mechanism offering the modified version is that it not only increases the spectrum of antimicrobial activity but also reduces hemolysis and allergic responses. ${ }^{8}$ These self-assembled AMPs might be the next generation antibiotics, thanks to their rapid and improved activity, which will make it more difficult for pathogenic microorganisms to achieve fast resistance. ${ }^{7}$

Bacitracin and gramicidin $S$ are bactericidal peptide antibiotics. Bacitracin, derived from Bacillus subtilis, inhibits the growth of other Gram-positive bacteria by interfering in cell wall synthesis. Gramicidin S, a derivative of gramicidin, produced by Bacillus brevis, is effective against both Gram-positive and Gram-negative bacteria as well as against some fungi, but offers hemolysis at an even lower concentration that is required for bacterial cell death, rendering it unsuitable for application. In this paper, we report the preparation of self-assembled nanofiber structure from AMPs, bacitracin and gramicidin S with modified action, which proved its application as a successful antibacterial ointment.

\section{RESULTS AND DISCUSSION}

Formation of self-assembled structure of bacitracin and gramicidin $S$ has been confirmed using CD spectroscopy and scanning electron microscopy (SEM) analyses. Examination of antimicrobial activity of the self-assembled peptides was carried out against both Grampositive and Gram-negative bacteria of quality control strains as well as hospital-isolated pathogenic strains. Cellular toxicity and hemolytic activity of the developed structures were also evaluated. Chloroform solutions of each peptide were prepared at a concentration of $100 \mathrm{mg} \mathrm{ml}^{-1}$ at $\mathrm{pH}$ 7.0. Bacitracin $\left(10 \mathrm{mg} \mathrm{ml}^{-1}\right)$ and gramicidin $\left(10 \mathrm{mg} \mathrm{ml}^{-1}\right)$ were rotated separately and combined in chloroform solution for $12 \mathrm{~h}$. The amount of bacitracin molecule remained unchanged $\left(10 \mathrm{mg} \mathrm{ml}^{-1}\right)$ and the gramicidin $\mathrm{S}$ was varied (1-10 $\left.\mathrm{mg} \mathrm{ml}^{-1}\right)$ to optimize the minimum requirement of gramicidin $\mathrm{S}$ to reduce the toxicity level. It was observed that bacitracin $\left(10 \mu \mathrm{g} \mathrm{ml}^{-1}\right)$ with gramicidin $\mathrm{S}\left(2 \mu \mathrm{g} \mathrm{ml}^{-1}\right)$ is the best combination to achieve broad-spectrum activity and less toxicity. Gramicidin $S$ and bacitracins are in the class of cyclic decapeptides with several hydrogen bonding sites in their outer core region, and they selfassociate individually in chloroform solutions yielding a helical $\beta$-sheet structure between them (Figure 1).

\footnotetext{
${ }^{1}$ Mass Spectrometry \& Proteomics Lab, Central Research Facility, Indian Institute of Technology, Kharagpur, Kharagpur, India and ${ }^{2}$ Centro de Análises Proteômicas e Bioquímicas, Programa de Pós-Graduação em Ciências Genômicas e Biotecnologia, UCB, Brasília, Brazil

Correspondence: Professor OL Franco, Centro de Análises Proteômicas e Bioquímicas, Programa de Pós-Graduação em Ciências Genômicas e Biotecnologia, UCB, SGAN 916N, Modulo B, Sala 219, Brasilia 70790-160, Brazil.

E-mail: ocfranco@gmail.com
}

Received 4 September 2013; revised 24 March 2014; accepted 28 April 2014; published online 4 June 2014 
A CD spectrum of self-assembled bacitracin shows a negative band at $212 \mathrm{~nm}$ and $194 \mathrm{~nm}$ along with a positive band at $205 \mathrm{~nm}$ indicative of $\beta$-sheet structure. Whereas self-assembled gramicidin $\mathrm{S}$ exhibited a minimum in the range of 230-232 nm typically representing aromatic stacking of two adjacent phenylalanine residues of the peptides. Moreover, positive bands at 220 and $205 \mathrm{~nm}$ along with a broad negative band at $212 \mathrm{~nm}$ are also indicative of random-coil $\beta$-sheet structure. Therefore, the mixture of bacitracin and gramicidin $\mathrm{S}$, based on the maxima 202 and $220 \mathrm{~nm}$ with minima at $212 \mathrm{~nm}$,

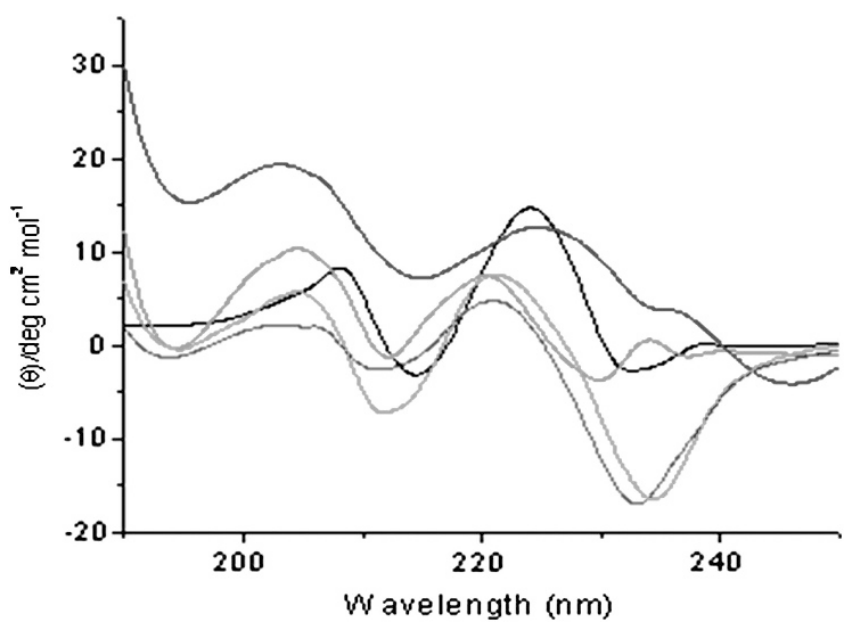

Figure $1 \mathrm{CD}$ spectra of the peptides rendering nanotubular structures after assembling. The line graph indicates only bacitracin (black line), bacitracin after $12 \mathrm{~h}$ rotation (red line), gramicidin S (blue line), gramicidin $\mathrm{S}$ after $12 \mathrm{~h}$ rotation (green line) and bacitracin-gramicidin S mixture rotation (pink line). A full color version of this figure is available at The Journal of Antibiotics journal online. is more a $\beta$-turn with some random coil nature because of a minimum at $194 \mathrm{~nm}$. Moreover, SEM image explored the left-handed coiled helical $\beta$-sheet morphology with $70 \mathrm{~nm}$ diameter (Figure 2) of self-assembled peptide mixture.

That yielded a nanotubular structure containing two already assembled peptides. From the MICs in Table 1, it is 32 -fold lower than that of bacitracin, 8-fold lower than that of self-assembled bacitracin, 4-fold lower than the MIC of gramicidin S and 2-fold lower than the MIC of self-assembled gramicidin S. AMPs, bacitracin and gramicidin $S$ are bactericidal by interfering in cell wall and phospholipid synthesis, respectively. Although gramicidin S offers higher and broader activity than bacitracin, toxicity is the major drawback, rendering it unsuitable for administration. Interestingly, the self-assembled ointment developed here revealed no significant change in cell cycle (data not shown) and its morphology was observed (Figure 3). Finally, the antimicrobial activities of the peptides are considered to increase owing to the formation of self-assembled nanostructure, whereas toxicity is reduced via blocking

Table 1 Minimum inhibitory concentration of antibiotics evaluated against multiple bacterial pathogens

\begin{tabular}{lcccc}
\hline Compounds & $\begin{array}{c}\text { Staphylococcus } \\
\text { epidermidis }\end{array}$ & $\begin{array}{c}\text { Escherichia } \\
\text { coli }\end{array}$ & $\begin{array}{c}\text { Pseudomonas } \\
\text { aeruginosa }\end{array}$ & $\begin{array}{c}\text { Staphylococcus } \\
\text { aureus }\end{array}$ \\
\hline Bac & 32 & nd & nd & 32 \\
Bac, 12 h & 8 & nd & nd & 8 \\
Gram & 4 & 4 & 4 & 4 \\
Gram, 12h & 2 & 2 & 2 & 2 \\
Bac + Gram, 12h & 1 & 1 & 1 & 1 \\
\hline
\end{tabular}

Abbreviations: Bac, Bacitracin; Gram, gramicidin; nd, not detected. Data are represented as $\mathrm{mgl}^{-1}$ concentration.
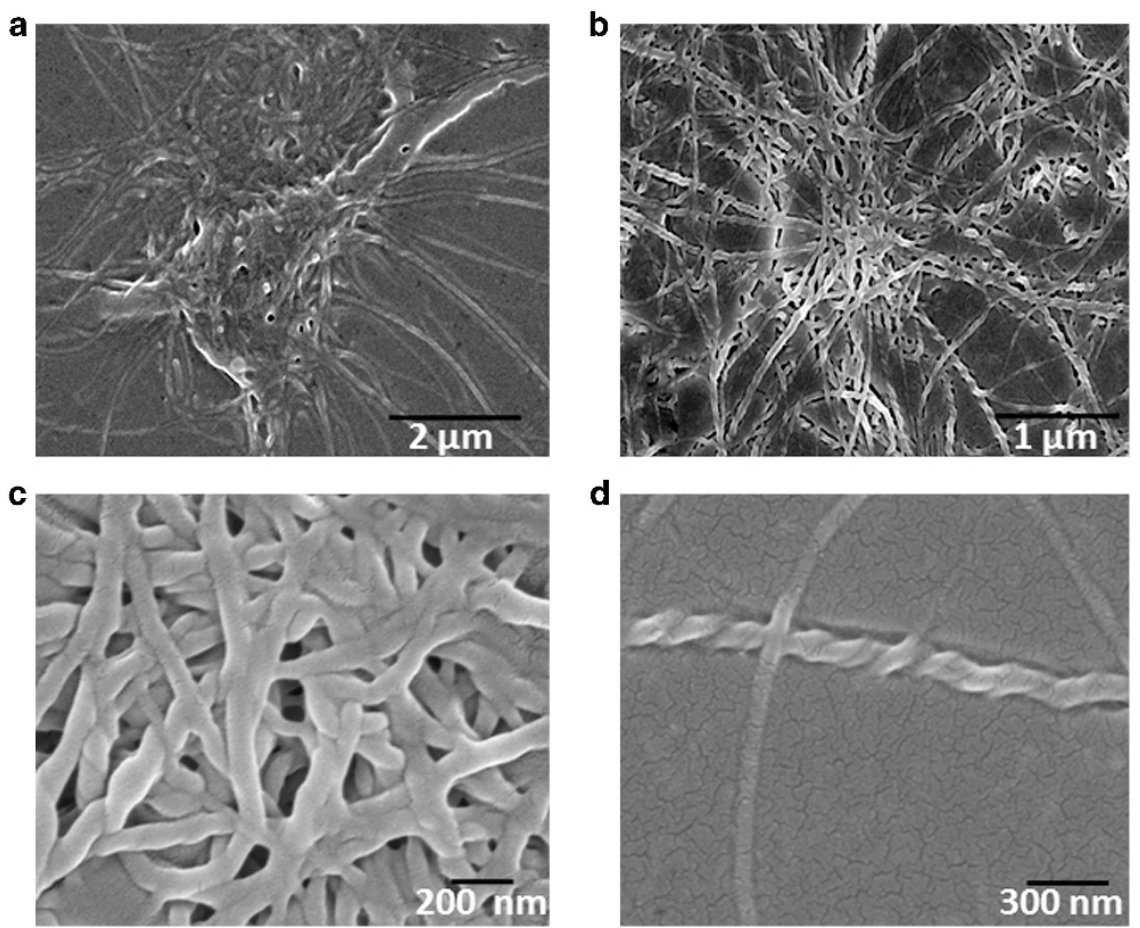

Figure 2 SEM images of self-assembled nanostructures formed after gramicidin S and bacitracin (after $12 \mathrm{~h}$ rotation) in chloroform solutions. 
a

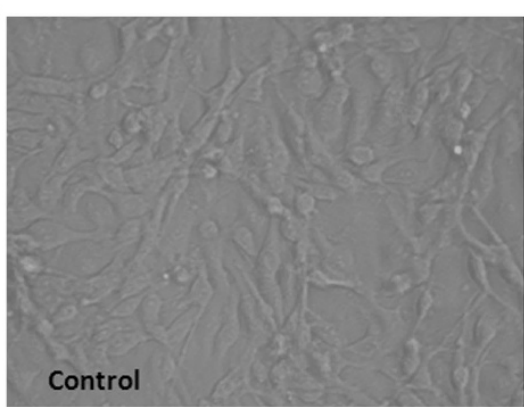

C

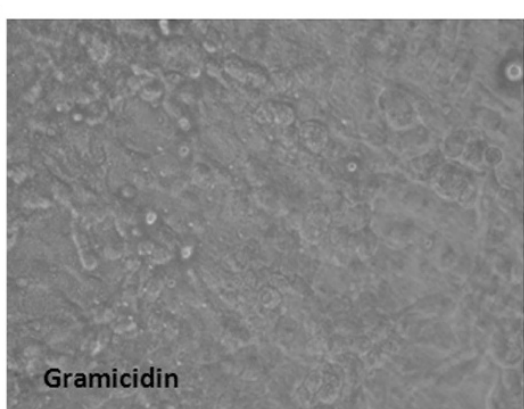

b

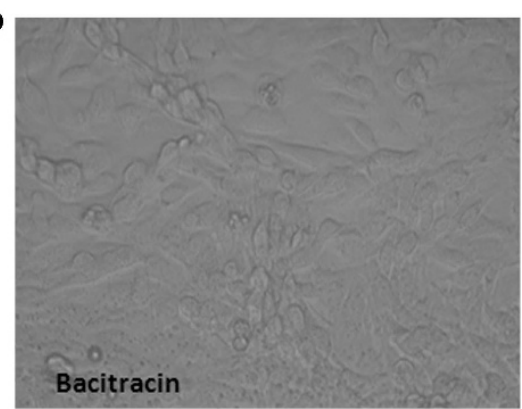

d

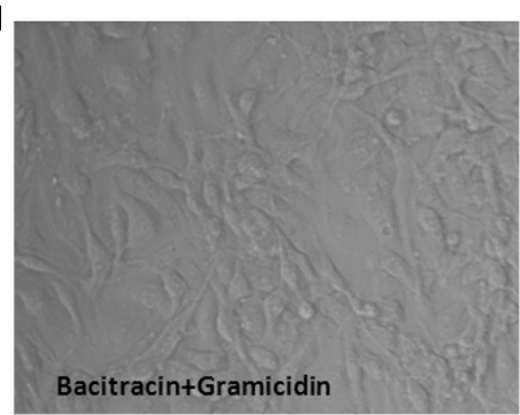

Figure 3 Representative optical microscopic images of MDA-MB-231 cells at $\times 40$ magnification. (a) Images are captured from well without any peptide treatment; (b) treated with bacitracin at $10 \mathrm{mgl}^{-1}$; (c) treated with gramicidin $\mathrm{S}$ at $10 \mathrm{mgl}^{-1}$; (d) treated with developed ointment. A full color version of this figure is available at The Journal of Antibiotics journal online.

of active functional moiety through strong hydrogen bonding interaction and the loss of toxicity function might be probable due to decrease in exposed positive charges, which are likely to be involved in the attachment of peptides to mammalian membrane surface, preventing cellular disruption. Therefore it can be used as a suitable ointment for infection control.

To evaluate and complement the in vitro results, theoretical models of gramicidin S and bacitracin were constructed. Procheck summary of bacitracin showed that $100 \%$ of amino-acid residues are located in the most favorable regions in the Ramachandran plot. Gramicidin S structure presented a ring with unusual amino-acid residues exposed as $\mathrm{Orn}^{2}, \mathrm{Orn}^{7}, \mathrm{Dpn}^{4}$ and $\mathrm{Dpn}^{9}$ (Figure 4a). Bacitracin presented a short $\alpha$-helix inside of ring after cyclization and addition of the carbonic chain $\left(\mathrm{C}_{6} \mathrm{H}_{12} \mathrm{~N}\right)$. Furthermore, this cyclization generates an 'amphipathic ring-tail peptide' with $36 \%$ of hydrophobic ratio and zero charge for the polypeptide chain. The amino-acid residues exposed in the 'amphipathic ring-tail peptide' were $\mathrm{Lys}^{6}$ and $\mathrm{His}^{9}$ on one side and $\mathrm{Ile}^{4}, \mathrm{Ile}^{7}$ and $\mathrm{Phe}^{8}$ on the other (Figure $4 \mathrm{~b}$ ). Hydrophobic amino-acid residues $\mathrm{Val}^{1}, \mathrm{Leu}^{3}, \mathrm{Val}^{6}$ and $\mathrm{Leu}^{8}$ seem to be important in the interaction for peptide oligomerization (Figure 4c).

Structural differences between the template structures and predicted three-dimensional structure of the peptide model were calculated by superimposing backbones onto the template structures. The root-mean-square deviation values for bacitracin between templates and theoretical models were $2.10 \AA$. In addition, the general quality for the model was reliable in according with a value of -0.21 for the g-factor. The root-mean-square deviation values and variability observed among the experimental structure templates and the modeled structure demonstrated a fold modification due to the postmodification in the structure of bacitracin.

The docking interaction analysis showed that gramicidin $S$ can form a stable trimer and starting with several trimers evolves to one big ring; however, computational power is not yet enough to construct a fiber (Figure 4). Otherwise, the study demonstrated that it is possible to form a fiber when we consider the free energy observed in trimers. However, the assembly for bacitracin and mixture are being studied in silico. At the moment these two situations have been studied by in-computational docking, but the energy observed in both systems is unreliable mainly due to the molecular complexity. The affinity observed in in silico studies for the mixture revealed that the cyclic molecules bacitracin and gramicidin $\mathrm{S}$ might interact in many different spatial positions, preventing the attainment of a favorable free energy. The complementarity observed between gramicidin's $\mathrm{S}$ interactions is favorable and it might be reinforced due to fit observed among electrostatic and hydrogen bond interactions, as gramicidin is composed of two identical semicircles that are inverted, so its molecules promote a perfect fit. All interactions observed were $<4.0 \AA$, but only the interactions around $3.6 \AA$ were highlighted (Figure $4 \mathrm{c}$ ). The interaction between the gramicidin $\mathrm{S}$ at central position in trimer formation involved the side chain $\mathrm{Leu}^{3}$ carbons (CD1) with the carbon side chain of $\mathrm{Leu}^{3}$ (CD2) of gramicidin S positioned at left side with distance of $3.6 \AA$. Another interaction observed occurred between the carbons (CD1) of $\mathrm{Val}^{1}$ side chain with the carbon (CD2) of $\mathrm{Leu}^{3}$ side chain. In another structural side, the interaction occurs between the nitrogens (NE) of $\mathrm{Orn}^{2}$ and $\mathrm{Orn}^{7}$ (NE) with the oxygen (O) of $\mathrm{Dpn}^{4}$ with distances of 3.2 and $3.1 \AA$, respectively. This information demonstrated that the peptide fits in the head-tail direction, probably organizing the central hydrophobic residues into the central core and exposing the charged residues in the poles.

\section{CONCLUSIONS}

In summary, the bacitracin and gramicidin S-based self-assembled nanopeptide ribbon may be a successful ointment formulation for bacterial infection control. Moreover, such fibers could be used in the future to construct novel material that could be used to control bacterial infection in hospitals. 


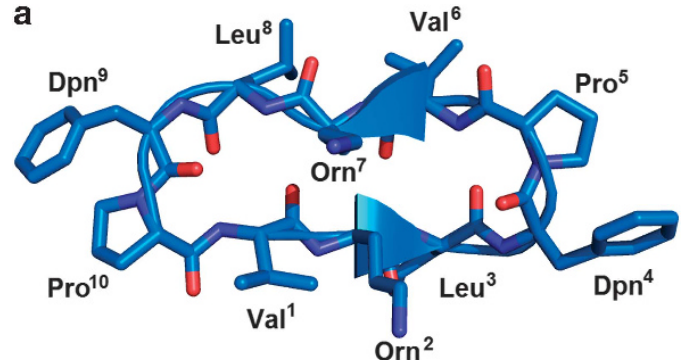

b

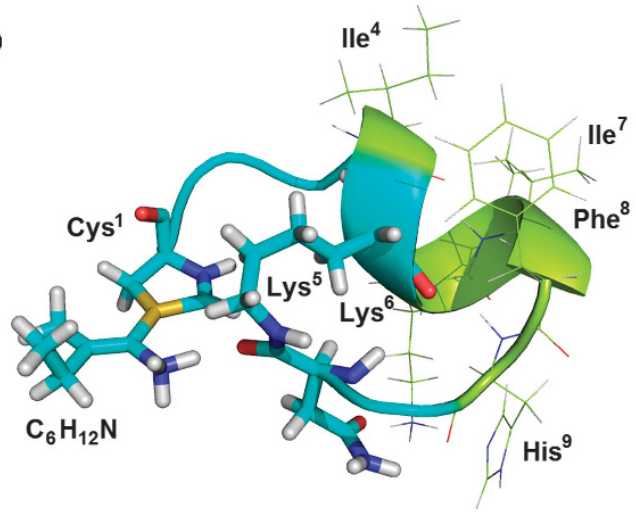

c

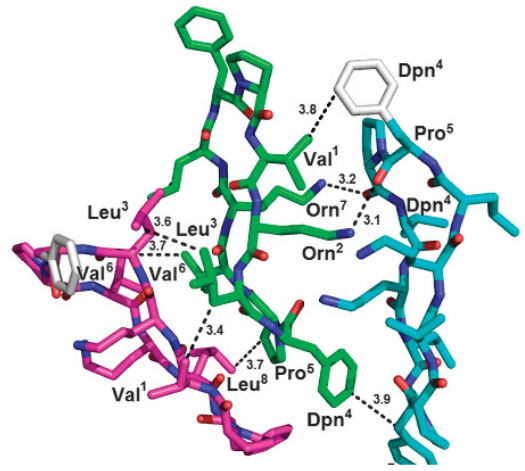

Figure 4 Tridimensional structure of bacitracin and gramicidin $\mathrm{S}$. (a) Bacitracin amino-acid residues of cyclopeptide. (b) Gramicidin S aminoacid residues involved in self-assembly. (c) Trimer formation of the peptide gramicidin S showing the structural complementarities among the cyclic peptides. Amino-acid residues probably interact stabilizing the complex forming non-covalent interactions (dotted lines)

\section{MATERIALS AND METHODS}

\section{Scanning electron microscopy (SEM)}

Aliquots $(100 \mu \mathrm{l})$ of each gel were prepared and placed in a 24 -well plate. Fiber was then affixed to SEM pucks using conductive carbon tape. The pucks were sputter-coated with $10-15 \mathrm{~nm}$ gold using a CRC-150 sputter-coater and imaged using an FEI Quanta 400 FESEM (Clayton South, VIC, Australia) at $20.00 \mathrm{kV}$.

\section{Determination of secondary structure by CD spectroscopy}

CD spectra were recorded using a Jasco-810 spectropolarimeter (JASCO Corporation, Hachioji City, Japan). For non-gelled peptides, samples at 1 wt $\%$ and $\mathrm{pH} 7$ were placed in a quartz cuvette with a path length of $0.001 \mathrm{~cm}$. For samples gelled with $\mathrm{Mg}^{2+}$, the resulting $1 \mathrm{wt} \%$ gel at $\mathrm{pH} 7$ was carefully pipetted into a $0.001 \mathrm{~cm}$ path length quartz cuvette, making sure to avoid bubble formation. Spectra were recorded at room temperature from 250 to $180 \mathrm{~nm}$, with a $0.2-\mathrm{nm}$ data pitch and a scan rate of $50 \mathrm{~nm} \mathrm{~min}^{-1}$. Millidegrees of rotation were converted to molar residual ellipticity.

\section{Cell viability assay}

For 2,3-bis[2-methoxy-4-nitro-S-sulfophenynl]H-tetrazolium-5 carboxanilide inner salt (XTT) assays, MDA-MB-231 cells were grown in 96-well plates overnight and all assays were conducted in growth medium with full serum and additives. For studies with free drugs, $10 \mathrm{mmoll}^{-1}$ stocks were serially diluted in dimethyl sulfoxide and then further diluted into the medium to give the appropriate concentration while minimizing precipitation associated with serial diluting in medium alone. For studies with nanogels, the nanoparticles were diluted in serum-free medium and added to the cells at $4{ }^{\circ} \mathrm{C}$ for $20 \mathrm{~min}$, washed to remove the particles and the cells returned to $37^{\circ} \mathrm{C}$ in complete medium with $10 \%$ fetal bovine serum for an additional $72 \mathrm{~h}$. Inhibitors were added and removed as mentioned earlier or left in the medium for $72 \mathrm{~h}$. Cell viability was quantified at $450 \mathrm{~nm}$ after the addition of $1 \mathrm{mg} \mathrm{ml}^{-1}$ XTT solution (Sigma-Aldrich, Bangalore, India) in phenol red-free Dulbecco's modified Eagle's medium containing phenoxy-methosulfate (Sigma-Aldrich). Dose-response curves were plotted by using GraphPad Prism software and $\mathrm{EC}_{50}$ values were calculated using this program.

\section{Bacterial strains and determination of MIC}

Two human pathogenic strains, Gram-positive Staphylococcus aureus and Gram-negative Escherichia coli, were collected from Microbial Type Culture Collection and Gene Bank Chandigarh and stored at $-80{ }^{\circ} \mathrm{C}$ in $40 \%$ (v:v) glycerol before use. These strains were cultured according to their specifications. The MICs of the hydrogel/nanofiber against the bacterial strains were measured by the broth microdilution method in accordance with the National Committee for Clinical Laboratory Standards method. MIC was determined twice, each time in triplicate, in 96-well plates. Briefly, strains were inoculated from fresh plates and grown overnight with shaking at 250 r.p.m. The cells were harvested by centrifugation and resuspended in Hilton broth to a final concentration of $10^{7} \mathrm{CFU} \mathrm{ml}{ }^{-1}$, as determined with a spectrophotometer at $600 \mathrm{~nm}$. Hydrogel was serially diluted on the plate. Standardized bacterial suspension $(100 \mathrm{ml})$ was added to each well. Positive controls contained no peptide. The plates were incubated for $18 \mathrm{~h}$ at $27^{\circ} \mathrm{C}$, and the lowest concentration of the hydrogel/fiber at which it prevented the growth of the bacteria (indicated by lack of visible turbidity) was recorded. Positive growth controls demonstrated visible turbidity after $18 \mathrm{~h}$ at $27^{\circ} \mathrm{C}$.

\section{Hemolytic assays}

Peptide hydrogels at different weight percentages $(0.5,1,1.5$ and $2 \mathrm{wt} \%)$ were prepared in a 96-well tissue culture polystyrene plate late as described above for the antibacterial assays. The hemolytic activity of the hydrogels was determined using human red blood cells (hRBCs). Blood was obtained for the study. The hRBCs were washed three times with bis-tris propane (BTP) buffer $(50 \mathrm{~mm}$, $150 \mathrm{~mm} \mathrm{NaCl}, \mathrm{pH} 7.4$ ) by centrifugation for $10 \mathrm{~min}$ at 3460 r.p.m. and $4{ }^{\circ} \mathrm{C}$ Then, $30 \mathrm{ml}$ of hRBCs was suspended in $12 \mathrm{ml}$ of BTP buffer $(50 \mathrm{~mm}, 150 \mathrm{~mm}$ $\mathrm{NaCl}, \mathrm{pH} 7.4$ ) resulting in a $0.25 \%$ (v:v) suspension. Subsequently, $80 \mathrm{ml}$ of the $\mathrm{hRBC}$ stock solution was introduced to individual tissue culture polystyrene plate control and hydrogel surfaces, followed by the addition of $170 \mathrm{ml}$ of BTP buffer to the top of each well for a final volume of $250 \mathrm{ml}$ corresponding to $0.08 \%$ (v:v) hRBCs. Samples were then incubated at $37^{\circ} \mathrm{C}$ with agitation for $1 \mathrm{~h}$. After which time, the solution at the top of the surfaces was removed and centrifuged at 14000 r.p.m. for $10 \mathrm{~min}$ at $4{ }^{\circ} \mathrm{C}$. Hemolytic activity was assessed by determining the amount of hemoglobin that was released as a result of cell membrane rupture. The release of hemoglobin was measured via the absorbance of the supernatant at $415 \mathrm{~nm}$. Controls for 0 and $100 \%$ hemolysis were defined by adding BTP buffer and a $1 \%$ Triton X-100 solution to hRBCs on the control surface, respectively. Each assay was performed in triplicate.

\section{Molecular modeling}

The three-dimensional model for gramicidin S was obtained in Protein Data Dank, pdb code 1tk2, which represents the crystal structure of the complex formed between alkaline proteinase savinase and gramicidin $S$ at $1.5 \AA .{ }^{9}$ Bacitracin was constructed in agreement with a previous study based on the structure of $1 \mathrm{p} 68$, which presented $46 \%$ of identity. ${ }^{10}$ The $1 \mathrm{p} 68$ structure used as template for bacitracin, named S-824, is a four-helix bundle domain from E. coli obtained through a combinatorial library of de novo amino-acid sequences. ${ }^{11}$ 
Two hundred theoretical tridimensional peptide structures were constructed using Modeller v.9.8 for bacitracin. ${ }^{12}$ Bacitracin final model was evaluated, that is, geometry, stereochemistry and energy distributions in the models, by using PROSA II to analyze packing and solvent exposure characteristics and PROCHECK for additional analysis of stereochemical quality. ${ }^{13}$ In addition, root-mean-square deviation was calculated by overlap of $\mathrm{C} \alpha$ traces and backbones on to the template structure through the program 3DSS. ${ }^{14}$ The peptide structures were visualized and analyzed on Delano Scientific's PYMOL ${ }^{15}$ (http://pymol.sourceforge.net/). In contrast, all combinatory conformation for constructing the trimers was not possible due to the lack of affinity and complementarities observed. This fact might be explained by the absence of hydrogen, electrostatic and hydrophobic bonds formation.

\section{In silico construction fiber with gramicidin $S$ and bacitracin peptides}

All docking calculations were performed using AUTODOCK 4.2 program. ${ }^{16}$ Docking simulation of both peptides (gramicidin $S$ and bacitracin) was performed toward itself and several combinatory possibilities. The fiber study was carried out with the construction of trimer combination with both peptides: first cluster, only a trimer composed of gramicidin S; second cluster, only a trimer composed of bacitracin; third cluster was a combination of two gramicidins S and one bacitracin; and fourth cluster was a combination of two bacitracin and one gramicidin S. All hydrogen atoms were added using the AutoDockTool. Grid maps were calculated with $35 \times 30 \times 25$ points to gramicidin $\mathrm{S}$ and bacitracin, and $1.0 \AA$ spacing centered. A Lamarckian genetic algorithm was used as the search method to find the best peptidepeptide complex. Fifty docking runs were carried out for all possibilities of combination. The generated structures were ranked in two steps: first a cluster with the best models with lowest energy and second with a root-mean-square deviation, for all atoms docked with the membrane, showing tolerance of $4 \AA$, as recommended for blind docking. ${ }^{17}$ The program $\mathrm{PyMol}^{15}$ (http://pymol. sourceforge.net/) was used to characterize peptide-membrane interactions.

\section{ACKNOWLEDGEMENTS}

This study was supported by grants from the CNPq, CAPES, FAPDF, EMBRAPA and UCB.
1 Falagas, M. E., Grammatikos, A. P. \& Michalopoulos, A. Potential of old-generation antibiotics to address current need for new antibiotics. Expert Rev. Anti Infect. Therapy 6, 593-600 (2008).

2 Zasloff, M. Antimicrobial peptides of multicellular organisms. Nature 415, 389-395 (2002).

3 Roy, A., Franco, O. L. \& Mandal, S. M. Biomedical exploitation of self assembled peptide based nano-structures. Curr. Protein Pept. Sci. 14, 580-587 (2013).

4 Anantharaman, A., Rizvi, M. S. \& Sahal, D. Synergy with rifampin and kanamycin enhances potency, kill kinetics, and selectivity of de novo-designed antimicrobial peptides. Antimicrob. Agents Chemother 54, 1693-1699 (2010).

5 Jenssen, H., Hamill, P. \& Hancock, R. E. Peptide antimicrobial agents. Clin. Microbiol. Rev. 19, 491-511 (2006).

6 Yenugu, S. \& Narmadha, G. The human male reproductive tract antimicrobial peptides of the HE2 family exhibit potent synergy with standard antibiotics. J. Pept. Sci. 16, 337-341 (2010).

7 Fernandez-Lopez, S. et al. Antibacterial agents based on the cyclic D,L-alpha-peptide architecture. Nature 412, 452-455 (2001).

8 Wang, H. et al. The efficacy of self-assembled cationic antimicrobial peptide nanoparticles against Cryptococcus neoformans for the treatment of meningitis. Biomaterials 31, 2874-2881 (2010).

9 Bhatt, V. S. et al. Crystal structure of the complex formed between alkaline proteinase savinase and gramicidin S at 1.5A resolution. Protein Data Bank http://www.ncbi.nlm. nih.gov/Structure/mmdb/mmdbsrv.cgi?uid=1tk2.

10 Mandal, S. M., Migliolo, L. \& Franco, O. L. The use of MALDI-TOF-MS and in silico studies for determination of antimicrobial peptides' affinity to bacterial cells. J. Am. Soc. Mass Spectrom. 23, 1939-1948 (2012).

11 Nicastro, G. et al. Solution structure of crotamine, a $\mathrm{Na}+$ channel affecting toxin from Crotalus durissus terrificus venom. Eur. J Biochem. 270, 1969-1979 (2003).

12 Wei, Y., Kim, S., Fela, D., Baum, J. \& Hecht, M. H. Solution structure of a de novo protein from a designed combinatorial library. Proc. NatI Acad. Sci. USA 100, 13270-13273 (2003).

13 Eswar, N. et al. Comparative protein structure modeling using Modeller. Curr. Protoc. Bioinformatics Chapter 5, Unit 5.6 (2006).

14 Sumathi, K., Ananthalakshmi, P., Roshan, M. N. \& Sekar, K. 3dSS: 3D structural superposition. Nucleic Acids Res. 34(Web Server issue), W128-W132 (2006).

15 DeLano, W. The PyMOL Molecular Graphics System (De Lano Scientific, San Carlos, CA, USA, 2002).

16 Sousa, S. F., Fernandes, P. A. \& Ramos, M. J. Protein-ligand docking: current status and future challenges. Proteins 65, 15-26 (2006).

17 Jitonnom, J., Lomthaisong, K. \& Lee, V. S. Computational design of peptide inhibitor based on modifications of proregion from Plutella xylostella midgut trypsin. Chem. Biol. Drug Des. 79, 583-593 (2012). 